\title{
Hemodynamics and myocardial blood flow patterns after placement of a cardiac passive restraint device in a model of dilated cardiomyopathy
}

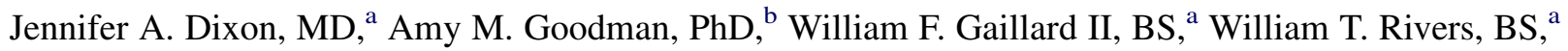

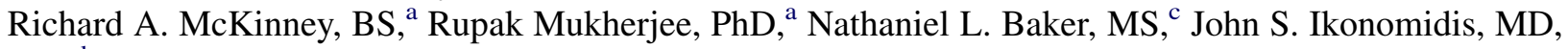 \\ $\mathrm{PhD},{ }^{\mathrm{b}}$ and Francis G. Spinale, $\mathrm{MD}, \mathrm{PhD}^{\mathrm{a}}$
}

\begin{abstract}
Background: The present study examined a cardiac passive restraint device which applies epicardial pressure (HeartNet Implant; Paracor Medical, Inc, Sunnyvale, Calif) in a clinically relevant model of dilated cardiomyopathy to determine effects on hemodynamic and myocardial blood flow patterns.
\end{abstract}

\begin{abstract}
Methods: Dilated cardiomyopatht was established in 10 pigs ( 3 weeks of atrial pacing, 240 beats $/ \mathrm{min}$ ). Hemodynamic parameters and regional left ventricular blood flow were measured under baseline conditions and after acute placement of the HeartNet Implant. Measurements were repeated after adenosine infusion, allowing maximal coronary vasodilation and coronary flow reserve to be determined.

Results: Left ventricular dilation and systolic dysfunction occurred relative to baseline as measured by echocardiography. Left ventricular end-diastolic dimension increased and left ventricular fractional shortening decreased ( $3.8 \pm 0.1$ vs $6.1 \pm 0.2 \mathrm{~cm}$ and $31.6 \% \pm 0.5 \%$ vs $16.2 \% \pm 2.1 \%$, both $P<.05$, respectively), consistent with the dilated cardiomyopathy phenotype. The HeartNet Implant was successfully deployed without arrhythmias and a computed median mid-left ventricular epicardial pressure of $1.4 \mathrm{~mm} \mathrm{Hg}$ was applied by the HeartNet Implant throughout the cardiac cycle. Acute HeartNet placement did not adversely affect steady state hemodynamics. With the HeartNet Implant in place, coronary reserve was significantly blunted.
\end{abstract}

Conclusions: In a large animal model of dilated cardiomyopathy, the cardiac passive restraint device did not appear to adversely affect basal resting myocardial blood flow. However, after acute HeartNet Implant placement, left ventricular maximal coronary reserve was blunted. These unique results suggest that cardiac passive restraint devices that apply epicardial transmural pressure can alter myocardial blood flow patterns in a model of dilated cardiomyopathy. Whether this blunting of coronary reserve holds clinical relevance with chronic passive restraint device placement remains unestablished. (J Thorac Cardiovasc Surg 2011;142:1038-45)

In the past decade the number of patients discharged from the hospital with a diagnosis of congestive heart failure (CHF) has doubled and CHF is now the most common reason for hospital admission in the elderly patient population. As the population ages, the incidence of CHF will increase and continue to be a major health care issue. The progression of CHF is characterized by left ventricular (LV) dilation and subsequent pump dysfunction. Current pharmacologic therapies for CHF include modulation of critical neurohormonal pathways that be-

From the Division of Cardiothoracic Surgery, ${ }^{\text {a }}$ Medical University of South Carolina and Ralph H. Johnson Veterans Affairs Medical Center, Charleston, SC; Paracor

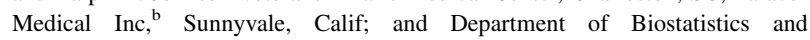
Epidemiology, ${ }^{\mathrm{c}}$ Medical University of South Carolina, Charleston, SC.

This research was supported by a grant from Paracor Medical, Inc, as well as by National Institutes of Health grants HL81692 and HL87134 and a Merit Award from the Veterans' Affairs Health Administration.

Received for publication Jan 12, 2010; revisions received Aug 13, 2010; accepted for publication Sept 9, 2010; available ahead of print March 14, 2011

Address for reprints: Francis G. Spinale, MD, PhD, Cardiothoracic Surgery, Strom Thurmond Research Center, 114 Doughty St, Suite 625, Charleston, SC 29425 (E-mail: wilburnm@musc.edu).

0022-5223/\$36.00

Copyright (C) 2011 by The American Association for Thoracic Surgery doi: $10.1016 /$ j.jtcvs.2010.09.065 come activated during progression of the disease. These pharmacologic treatment strategies may provide symptomatic relief, but the LV remodeling process can continue unabated. Surgical treatment for LV dilation and systolic dysfunction has also been pursued, including the use of cardiac support devices that are postulated to attenuate progressive dilation and stabilize systolic function. ${ }^{1-7}$ For example, placement of passive cardiac support devices in patients with dilated cardiomyopathy (DCM), a classic phenotypic presentation of LV dilation and CHF, has been performed with an aim of altering the course of $\mathrm{CHF}^{1-3,5,6}$ However, a number of issues surrounding the deployment of these devices in the context of DCM remain. Specifically, the acute hemodynamic consequences, and more important the direct effect on myocardial blood flow patterns with acute placement of a cardiac support device, remain poorly understood. A particular passive support device that has advanced to clinical trials is the HeartNet Implant (model P140; Paracor Medical, Inc, Sunnyvale, Calif), which consists of silicone-insulated nitinol wire forms. The HeartNet Implant provides resistance to circumferential dilation via transmural myocardial pressure..$^{5-7}$ In the context of DCM, 


\section{Abbreviations and Acronyms \\ $\mathrm{CHF}=$ congestive heart failure \\ $\mathrm{DCM}=$ dilated cardiomyopathy \\ $\mathrm{ECG}=$ electrocardiogram (graphy) \\ $\mathrm{LV}=$ left ventricle (ventricular) \\ $\mathrm{RV} \quad=$ right ventricle (ventricular)}

a shift in the volume/wall thickness ratio occurs, whereby changes in epicardial pressure may directly affect intrinsic myocardial resistance to blood flow. These changes in pressure may be of particular relevance considering the HeartNet Implant may exert additive transmural compressive forces during diastole, the peak of myocardial blood flow delivery. However, whether this theoretical concern can be demonstrated under physiologic conditions relevant to DCM remains unknown. Accordingly, the present study used a large animal model that recapitulates the phenotype of $\mathrm{DCM}^{8}$ to measure hemodynamics and myocardial blood flow patterns at rest and after maximal coronary artery dilatation in the context of HeartNet Implant placement.

\section{METHODS}

The study used a DCM model that has been previously characterized by this laboratory. ${ }^{8-15}$ After the development of DCM, a terminal study was performed at which time baseline systemic hemodynamics and myocardial blood flow were measured with and without the HeartNet Implant in place. Two models of the HeartNet system were used: the model P140 system, which is currently undergoing study in the PEERLESS-HF pivotal trial in the United States and Canada, and the model P150 system, which is a next-generation HeartNet system. All animals were treated and cared for in accordance with the National Institutes of Health's "Guide for the Care and Use of Laboratory Animals" (National Institutes of Health, 1996).

\section{DCM Induction and Echocardiography}

Yorkshire pigs ( $\mathrm{n}=10 ; 25 \mathrm{~kg}$ castrated males) underwent pacemaker implantation for the purposes of establishing pacing-induced DCM. In brief, a sterile left thoracotomy and a small pericardiotomy were performed, exposing the left atrium. A shielded pacemaker lead (CapSure 4965; Medtronic, Inc, Minneapolis, Minn) was sutured onto the left atrium and connected to a pacemaker (Telectronics model 1256; St Jude Medical, Inc, Sylmar, Calif), which was buried in a subcutaneous pocket. The pericardium was irrigated and approximated, the thoracotomy closed, and the pleural space evacuated of air. On postoperative day 5, baseline echocardiography was performed (Agilent Sonos 5500, 3.5 MHz probe; Agilent Technologies, Inc, Santa Clara, Calif) and the pacemaker was then activated (240 beats/min). A 1:1 rate of capture was verified via electrocardiographic (ECG) recordings and echocardiography. Cardiac auscultation and ECG recordings were performed frequently during the pacing protocol to ensure continuous capture. At 18 to 21 days of pacing, the animal was returned to the laboratory for pacemaker deactivation, and a second echocardiogram was performed.

\section{Hemodynamic and Myocardial Blood Flow Measurements}

For the terminal studies, all animals were instrumented in identical fashion. Anesthetic induction commenced using isoflurane $(3 \%)$ and nitrous oxide $(33 \%)$ delivered via face mask. A $2-\mu \mathrm{g} / \mathrm{kg}$ dose of sufentanyl (Elkins-Sinn, Inc, Cherry Hill, NJ), a 0.1-mg/kg dose of etomidate (Amidate; Elkins-Sinn, Inc), and a 10-mg dose of vecuronium (Bedford Laboratories, Bedford, Ohio) were then administered intravenously, and an endotracheal tube was placed. Anesthesia was maintained with the delivery of inhaled isoflurane $(0.5 \%)$ and intravenous morphine (Elkins-Sinn, Inc) at $3 \mathrm{mg} \cdot \mathrm{kg}^{-1} \cdot \mathrm{h}^{-1}$. A bolus of amiodarone $(5 \mathrm{mg} / \mathrm{kg}$ ) (APP Pharmaceuticals LLC, Schaumburg, Ill) was administered, an intravenous infusion of lidocaine (Hospira, Inc, Lake Forest, Ill) was initiated at $4 \mathrm{mg} \cdot \mathrm{kg}^{-1}$. $\mathrm{h}^{-1}$, and magnesium chloride ( $\left.1 \mathrm{~g}\right)$ (Luitpold Pharmaceuticals, Inc, Shirley, $\mathrm{NY}$ ) was delivered. After stabilization of the surgical anesthetic plane, an intravenous infusion of vecuronium was initiated at $15 \mathrm{mg} \cdot \mathrm{kg}^{-1} \cdot \mathrm{h}^{-1}$.

A multilumened thermodilution catheter (7.5F; Baxter Healthcare, Inc, Irvine, Calif) was positioned in the pulmonary artery via the left external jugular vein. An $8 \mathrm{~F}$ introducer with a sidearm was placed in the right carotid artery for blood pressure measurements and subsequent placement of the LV pressure/infusion catheter. A previously calibrated microtipped transducer catheter with a side port for infusion and a pigtail catheter (7.5F; Millar Instruments, Inc, Houston, Tex) were advanced through the hemostatic sheath and positioned in the LV. The left carotid artery was cannulated with an $8 \mathrm{~F}$ hemostatic introducer with a sidearm for arterial blood pressure measurements and withdrawal of reference blood samples. A sternotomy was performed and the pericardium was opened and supported by pericardial sutures before baseline measurements. After instrumentation and a 30-minute stabilization period, the experimental protocol was begun.

\section{Experimental Protocol}

Steady state hemodynamics, specifically heart rate, oxygen saturation, arterial blood pressure, pulmonary artery pressure, pulmonary capillary wedge pressure, LV pressure, and cardiac output, as well as myocardial blood flow, were recorded with the ventilator temporarily suspended to prevent respiratory artifact. LV peak positive $\mathrm{dP} / \mathrm{dt}$ was determined from the digitized LV pressure signal. After completion of the steady state hemodynamic measurements, a set of fluorescent microspheres was injected for the determination of myocardial blood flow. ${ }^{14-17}$ In brief, blue-green (excitation 430, emission 460), yellow (excitation 490, emission 520), red-orange (excitation 530, emission 570), or scarlet (excitation 645, emission 680) fluorescent microspheres $\left(3 \times 10^{6}\right.$; Molecular Probes, Eugene, Ore) were injected into the LV via the pigtail catheter. A reference aortic blood sample was withdrawn from the contralateral carotid artery at a rate of $7 \mathrm{~mL} / \mathrm{min}$, which was initiated 5 seconds before injection and continued for 120 seconds after injection. Next, adenosine $\left(1.5 \mu \mathrm{moL} \cdot \mathrm{kg}^{-1} \cdot \mathrm{h}^{-1}\right)$ was infused through the venous access port, and after 15 minutes of infusion, hemodynamics and LV pressures were recorded and fluorescent microspheres delivered. The adenosine infusion allowed for maximal coronary vasodilation and determination of maximal coronary blood flow reserve. $^{8,10}$ After microsphere delivery, the adenosine infusion was terminated, resulting in a rapid normalization of hemodynamics.

\section{HeartNet Implant Placement and Measurements}

After steady state baseline measurements, the HeartNet Implant was placed circumferentially around the LV and right ventricle (RV). Two versions of the HeartNet Implant were modified for porcine sizing and used for the studies described herein: the P140 HeartNet and the P150 HeartNet Implant. Both the P140 and P150 HeartNet Implants consist of a series of circumferentially oriented silicone-jacketed nitinol wire-form rings that provide elastic support to the ventricular wall (approximately 0.5-2.5 $\mathrm{mm} \mathrm{Hg}$ throughout the cardiac cycle). The modified implants consisted of 3 rows and were designed to fit a heart with a maximum circumference measuring approximately $40 \mathrm{~cm}$ in circumference. The animals received either P140 Implants $(n=5)$ or P150 Implants $(n=5)$ as determined by a preassigned randomization table. A demonstration of the intraoperative P140 Implant placement, assuring proper circumferential deployment, is shown in Figure 1, A. Furthermore, after visual confirmation of appropriate 


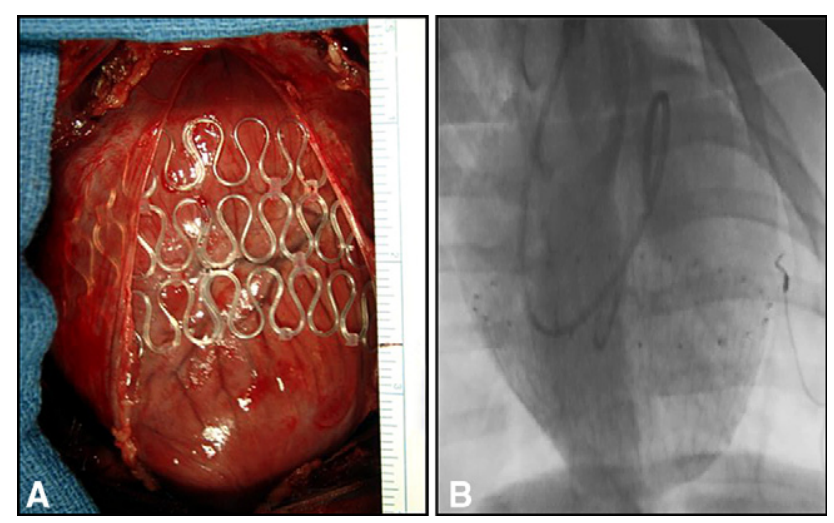

FIGURE 1. A, Intraoperative placement of the P140 Implant onto a DCM pig heart. B, Fluoroscopic image of the P140 Implant confirming circumferential deployment in the posterior region of the DCM heart. DCM, Dilated cardiomyopathy.

deployment, adequate posterior region placement was confirmed using fluoroscopic imaging (Phillips BV 300; Phillips Medical Systems, Bothell, Wash). A representative fluoroscopic image of the LV with the P140 Implant in place is shown in Figure 1, $B$. The diameters of the cardiac silhouette at the top circumferential ring of the HeartNet device changed from a baseline measurement of $244 \pm 4$ pixels to $240 \pm 4$ pixels (ratio of silhouette diameter after HeartNet placement to baseline diameter: $0.99 \pm 0.01$; $P=.82$ ). Once the HeartNet Implant had been placed, a second 30-minute stabilization period was observed, and then steady state hemodynamic measurements, LV pressure measurements, and fluorescent microsphere delivery were performed as described in the preceding section. After these measurements, an adenosine infusion was again instituted and the hemodynamic and coronary flow measurements were repeated.

\section{Tissue Harvest}

Isoflurane anesthesia was increased to $5 \%$ and, with a full surgical plane of anesthesia, the heart was harvested. The LV and RV were dissected free, weighed, and subsequently fixed in $10 \%$ formaldehyde to facilitate slicing. The myocardium was later dissected into $2 \mathrm{RV}$ regions and $18 \mathrm{LV}$ regions, including epicardial and endocardial regions. Samples of approximately $3 \mathrm{~g}$ each were collected from each region and digested in a potassium hydroxide solution as previously described. ${ }^{8-10,13,16,17}$ The aortic blood samples were extracted with an identical digest solution. The fluorescence of each extracted sample was subjected to spectrofluorimetry.

\section{Computations and Data Analysis}

Echocardiographic indices of LV end-diastolic dimension and fractional shortening were determined and compared between baseline prepacing values and DCM values using a $t$ test. From the hemodynamic data, pulmonary and systemic vascular resistances were computed from the thermodilution cardiac output and pressure measurements using standard formulas. The pulmonary artery waveforms recorded during determination of pulmonary capillary wedge pressure were analyzed to determine the amplitude of the a-wave (between the P-wave and the QRS complex on the ECG) and v-wave (coincident with the termination of the T-wave on the ECG) tracing before and after placement of the HeartNet devices. Regional blood flow computations were determined by the standard formula: $\mathrm{Qm}=(\mathrm{Ar} \times \mathrm{Am}) / \mathrm{Qr}$, where $\mathrm{Qm}$ is the blood flow in milliliters per minute, Ar is the fluorescence of the aortic reference sample, Am is the fluorescence of the tissue sample, and Qr is the withdrawal rate of the reference sample. ${ }^{20}$ Final blood flow values were normalized to tissue sample weights and expressed as milliliters per minute per gram. Comparisons of hemodynamics and myocardial blood flow response to adenosine, both with and without the HeartNet Implant placed, were made by repeated measures mixed model analysis of variance on each outcome variable, with grouping (baseline or HeartNet Implant) as the betweensubjects variable and drug status (steady state vs adenosine) as the within-subjects variable. Finally, the relative change from absolute baseline steady state values to values after HeartNet Implant placement and adenosine infusion were calculated and log transformed, inasmuch as they did not conform to a normal distribution. All pairwise comparisons were performed by Tukey adjusted $P$ values. In addition, potential HeartNet Implant/adenosine interactions were examined by analysis of variance. All statistical analyses were performed using the SAS statistical software (version 9.2; SAS Institute, Inc, Cary, NC) and statistical significance was considered at $\alpha=.05$ level. All data are presented as mean \pm standard error of the mean. All authors had access to the data and its interpretation and take full responsibility for its integrity. The funding agency did not influence the interpretation of the data.

\section{RESULTS}

Echocardiographic measures confirmed that the DCM phenotype was established by rapid atrial pacing and produced increased LV end-diastolic dimension $(3.8 \pm 0.1$ $\mathrm{cm}$ vs $6.1 \pm 0.2 \mathrm{~cm}$ ), decreased LV end-diastolic wall thickness $(0.8 \pm 0.1 \mathrm{~cm}$ vs $0.6 \pm 0.1 \mathrm{~cm})$, and decreased LV fractional shortening $(31.6 \% \pm 0.5 \%$ vs $16.2 \% \pm 2.1 \%)$, as compared with baseline values, consistent with prior reports from this laboratory and others..$^{8-14,18-20}$ The amplitudes of the a- and $\mathrm{v}$-waves on the pulmonary capillary wedge pressure traces were $18 \pm 3 \mathrm{~mm} \mathrm{Hg}$ and $22 \pm 3 \mathrm{~mm} \mathrm{Hg}$, respectively, suggesting the presence of at least mild and/ or moderate mitral regurgitation, consistent with the development of tachycardia-induced DCM. ${ }^{18-20}$ The hemodynamic effects observed with adenosine infusion while the HeartNet Implant was placed on the heart are reported in Table 1. Hemodynamics were equivalent at steady state and with adenosine in the pigs randomized to P140 or P150 HeartNet Implant and, therefore, were combined for all analyses for the purposes of statistical robustness. A decrease in systemic blood pressure was observed with adenosine administration, consistent with the systemic vasodilatory effects of adenosine. After acute placement of either the P140 or the P150 HeartNet Implant, steady state hemodynamics were unchanged from baseline steady state measurements. After HeartNet Implant placement, the amplitudes of the a- and v-waves on the pulmonary capillary wedge pressure tracing were similar to baseline values (a-wave: $18 \pm 3, P=.77$; v-wave: $21 \pm 3 \mathrm{~mm} \mathrm{Hg}$, $P=.61$, respectively), suggesting that there was minimal to no acute effect of HeartNet Implant placement on the degree of mitral regurgitation. Systemic blood pressure was reduced with adenosine infusion. Myocardial blood flow measurements at steady state and after adenosine, both with and without HeartNet Implant placement, are reported as LV endocardial, epicardial, or RV regional values (Table 2). Consistent with prior reports, myocardial blood flow was augmented by adenosine infusion in both chambers and in all regions analyzed. ${ }^{8-10}$ After acute HeartNet 
TABLE 1. Hemodynamics in DCM pigs under steady state and after adenosine at baseline and with placement of model P140 or P150 HeartNet Implants

\begin{tabular}{|c|c|c|c|c|}
\hline & \multicolumn{2}{|c|}{ Baseline } & \multicolumn{2}{|c|}{ HeartNet Implant } \\
\hline & Steady state & Adenosine & Steady state & Adenosine \\
\hline Heart rate (beats/min) & $101 \pm 7$ & $94 \pm 6$ & $108 \pm 7$ & $105 \pm 8$ \\
\hline \multicolumn{5}{|l|}{ LV pressures } \\
\hline LV peak (mm Hg) & $107 \pm 7$ & $95 \pm 5$ & $118 \pm 8$ & $103 \pm 8$ \\
\hline LVEDP (mm Hg) & $20 \pm 2$ & $21 \pm 3$ & $22 \pm 2$ & $22 \pm 3$ \\
\hline Peak positive $\mathrm{dP} / \mathrm{dt}(\mathrm{mm} \mathrm{Hg} / \mathrm{s})$ & $1229 \pm 113$ & $1163 \pm 105$ & $1461 \pm 137$ & $1332 \pm 108$ \\
\hline Peak negative $\mathrm{dP} / \mathrm{dt}(\mathrm{mm} \mathrm{Hg} / \mathrm{s})$ & $1626 \pm 120$ & $1388 \pm 122$ & $1540 \pm 102$ & $1444 \pm 124$ \\
\hline \multicolumn{5}{|l|}{ Systemic pressures } \\
\hline Systolic (mm Hg) & $109 \pm 6$ & $96 \pm 5$ & $120 \pm 8$ & $103 \pm 8$ \\
\hline Diastolic (mm Hg) & $69 \pm 5$ & $51 \pm 5^{*}$ & $80 \pm 7$ & $59 \pm 9 *$ \\
\hline Mean $(\mathrm{mm} \mathrm{Hg})$ & $86 \pm 6$ & $68 \pm 5^{*}$ & $98 \pm 8$ & $76 \pm 9^{*}$ \\
\hline $\operatorname{SVR}\left(\right.$ dyn $\left.\cdot \mathrm{s} \cdot \mathrm{cm}^{-5}\right)$ & $2542 \pm 273$ & $1714 \pm 267 *$ & $2767 \pm 223$ & $1887 \pm 373^{*}$ \\
\hline \multicolumn{5}{|l|}{ Pulmonary pressures } \\
\hline Systolic (mm Hg) & $32 \pm 3$ & $30 \pm 3$ & $35 \pm 2$ & $34 \pm 3$ \\
\hline Diastolic (mm Hg) & $24 \pm 3$ & $22 \pm 3$ & $27 \pm 3$ & $26 \pm 3$ \\
\hline Mean $(\mathrm{mm} \mathrm{Hg})$ & $28 \pm 3$ & $26 \pm 3$ & $31 \pm 3$ & $30 \pm 3$ \\
\hline PCWP $(\mathrm{mm} \mathrm{Hg})$ & $19 \pm 3$ & $19 \pm 3$ & $21 \pm 3$ & $23 \pm 3$ \\
\hline $\operatorname{PVR}\left(\right.$ dyn $\left.\cdot \mathrm{s} \cdot \mathrm{cm}^{-5}\right)$ & $361 \pm 76$ & $252 \pm 44$ & $386 \pm 68$ & $310 \pm 119$ \\
\hline Cardiac output (L/min) & $2.5 \pm 0.4$ & $2.8 \pm 0.4$ & $2.3 \pm 0.3$ & $2.7 \pm 0.4$ \\
\hline
\end{tabular}

TABLE 2. Myocardial blood flow in DCM pigs under steady state and after adenosine at baseline and with placement of model P140 or P150 HeartNet Implant

\begin{tabular}{|c|c|c|c|c|}
\hline \multirow[b]{2}{*}{ Tissue region } & \multicolumn{2}{|c|}{ Baseline } & \multicolumn{2}{|c|}{ HeartNet Implant } \\
\hline & Steady state & Adenosine & Steady state & Adenosine \\
\hline \multicolumn{5}{|l|}{ LV base } \\
\hline \multicolumn{5}{|l|}{ Free wall } \\
\hline Endocardium & $1.84 \pm 0.25$ & $4.14 \pm 0.73^{*}$ & $2.73 \pm 0.39$ & $3.15 \pm 0.38$ \\
\hline Epicardium & $1.89 \pm 0.21$ & $3.79 \pm 0.51 *$ & $3.02 \pm 0.43^{*}$ & $2.92 \pm 0.34$ \\
\hline \multicolumn{5}{|l|}{ Septal wall } \\
\hline Endocardium & $1.97 \pm 0.32$ & $3.71 \pm 0.56^{*}$ & $2.57 \pm 0.32$ & $3.09 \pm 0.52$ \\
\hline Epicardium & $1.94 \pm 0.39$ & $3.97 \pm 0.67 *$ & $2.64 \pm 0.41$ & $3.34 \pm 0.68$ \\
\hline \multicolumn{5}{|l|}{ LV mid } \\
\hline \multicolumn{5}{|l|}{ Free wall } \\
\hline Endocardium & $2.12 \pm 0.26$ & $4.54 \pm 0.78^{*}$ & $3.06 \pm 0.43$ & $3.64 \pm 0.53$ \\
\hline Epicardium & $2.14 \pm 0.27$ & $4.07 \pm 0.60 *$ & $3.19 \pm 0.42$ & $2.99 \pm 0.36$ \\
\hline \multicolumn{5}{|l|}{ Septal wall } \\
\hline Endocardium & $2.13 \pm 0.30$ & $3.84 \pm 0.68 *$ & $2.89 \pm 0.43$ & $3.27 \pm 0.64$ \\
\hline Epicardium & $2.18 \pm 0.35$ & $4.15 \pm 0.81 *$ & $3.01 \pm 0.50$ & $3.56 \pm 0.65$ \\
\hline \multicolumn{5}{|l|}{ LV apex } \\
\hline \multicolumn{5}{|l|}{ Free wall } \\
\hline Endocardium & $2.08 \pm 0.26$ & $4.00 \pm 0.64 *$ & $2.93 \pm 0.38$ & $3.27 \pm 0.41$ \\
\hline Epicardium & $2.03 \pm 0.28$ & $3.79 \pm 0.64 *$ & $2.86 \pm 0.42$ & $2.82 \pm 0.47$ \\
\hline \multicolumn{5}{|l|}{ Septal wall } \\
\hline Endocardium & $2.20 \pm 0.31$ & $4.12 \pm 0.84 *$ & $2.99 \pm 0.49$ & $3.49 \pm 0.68$ \\
\hline Epicardium & $1.95 \pm 0.24$ & $3.80 \pm 0.67 *$ & $2.85 \pm 0.45$ & $3.33 \pm 0.65$ \\
\hline \multicolumn{5}{|l|}{$\mathrm{RV}$} \\
\hline Free wall & $1.62 \pm 0.24$ & $3.80 \pm 0.65^{*}$ & $2.58 \pm 0.44$ & $3.55 \pm 0.89^{*}$ \\
\hline Outflow tract & $2.00 \pm 0.29$ & $3.89 \pm 0.68^{*}$ & $2.93 \pm 0.44$ & $3.61 \pm 0.72^{*}$ \\
\hline
\end{tabular}

Values are presented as mean \pm standard error of the mean. Blood flow is reported as milliliters per minute per gram of tissue. $D C M$, Dilated cardiomyopathy; $L V$, left ventricle; $R V$, right ventricle. Sample size: $\mathrm{n}=10 \mathrm{DCM}$ pigs. ${ }^{*} P<.05$ vs baseline steady state. 

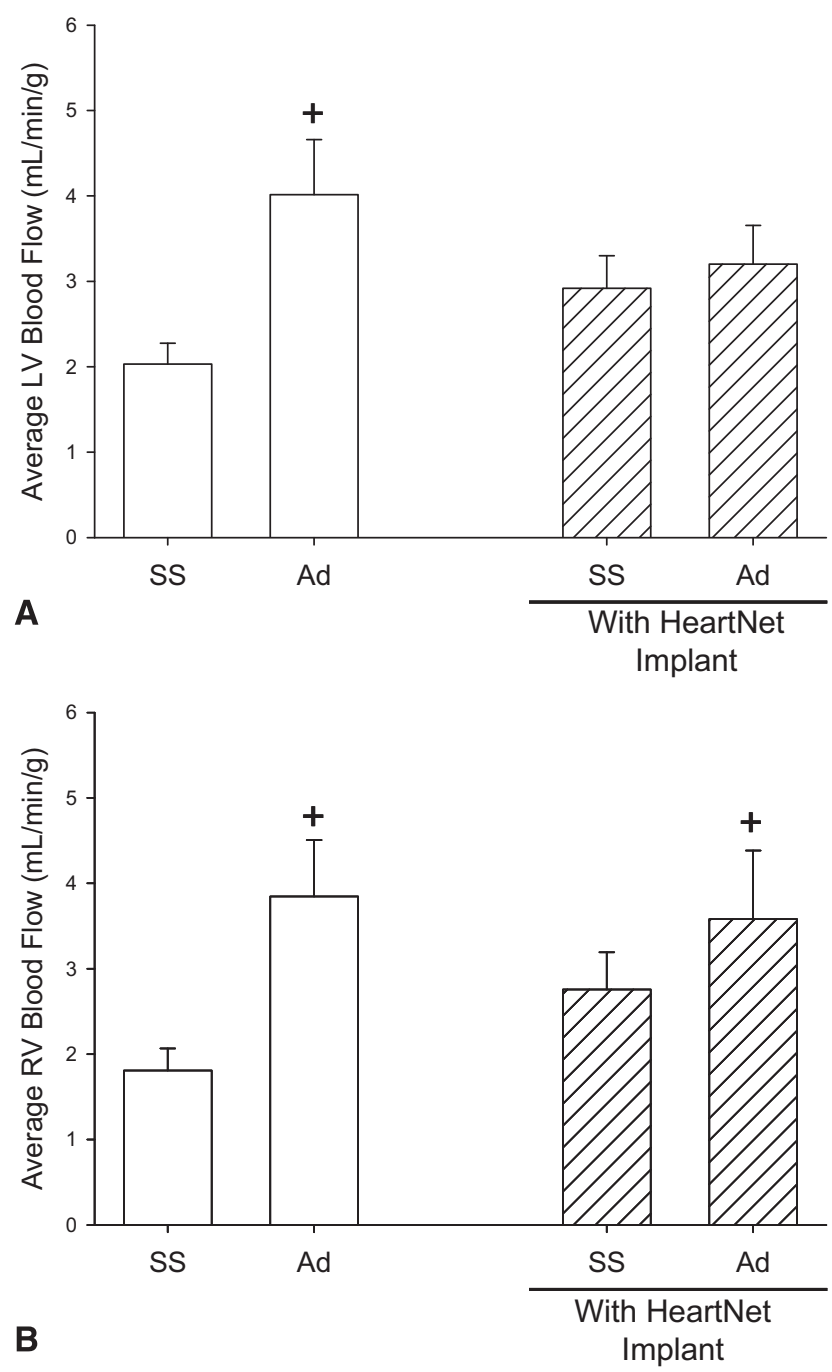

FIGURE 2. A, Average of left ventricular $(L V)$ blood flow from all measured regions in DCM pigs $(\mathrm{n}=10)$. A significant increase in LV blood flow values was observed under steady state conditions with adenosine administration. The effect of adenosine on LV blood flow is attenuated after acute HeartNet Implant placement. B, Average of right ventricular $(R V)$ blood flow from all measured regions in DCM pigs $(\mathrm{n}=10)$. Before and after acute HeartNet Implant placement, blood flow values increased from baseline steady state values with adenosine infusion. $+P<.05$ versus baseline steady state. $S S$, Steady state; $A d$, adenosine; $D C M$, dilated cardiomyopathy.

Implant placement, steady state myocardial blood flow increased from baseline steady state values and reached statistical significance in the LV base-free wall epicardial region. Moreover, after adenosine infusion, myocardial blood flow did not reach similar values as compared with the baseline adenosine state. Average LV and RV myocardial blood flow changes with and without HeartNet Implant are shown in Figure 2. Before HeartNet Implant placement, maximal coronary blood flow response, as assessed by adenosine infusion, increased from baseline
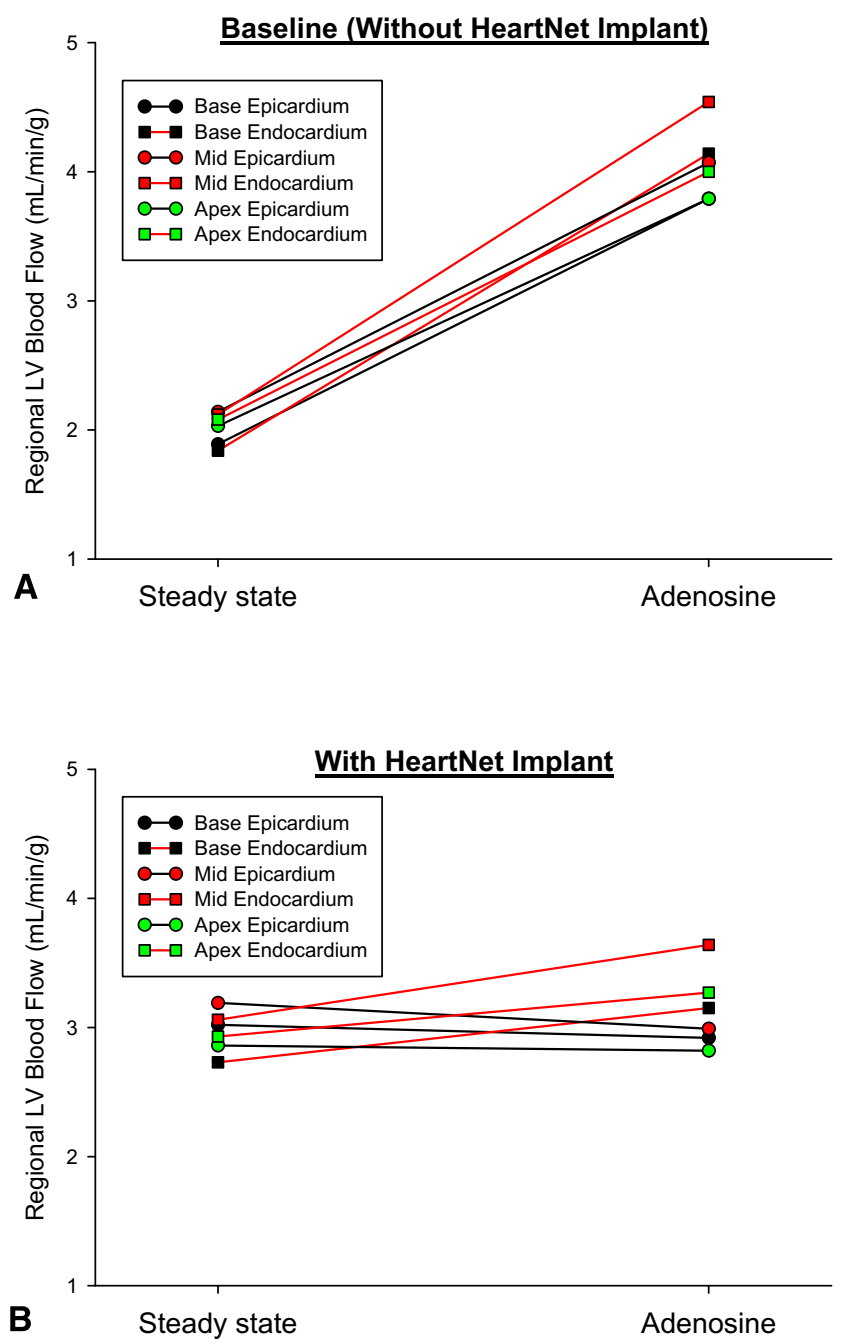

FIGURE 3. A, Average left ventricular $(L V)$ blood flow demonstrated by region at baseline under steady state conditions and after adenosine administration. Each region is the average blood flow from 10 DCM pigs. All LV free wall regions show a consistent increase in myocardial blood flow with adenosine administration. B, The same LV free wall regions, each the average of 10 DCM pigs, at steady state and with adenosine administration after HeartNet Implant placement. The 3 LV epicardial regions show a decrease in blood flow as compared with the LV endocardial regions after HeartNet Implant placement $(P=.02)$. $D C M$. Dilated cardiomyopathy.

steady state values. After acute HeartNet Implant placement, steady state LV myocardial blood flow values qualitatively increased but did not reach statistical significance. With HeartNet placement and adenosine infusion, LV myocardial blood flow did not significantly increase from either baseline steady state or HeartNet steady state. The average RV blood flow values, as shown in Figure 2, showed a similar pattern, but with a notable exception. Specifically, average RV myocardial blood flow increased from baseline steady state with adenosine infusion both before and after HeartNet Implant placement. 

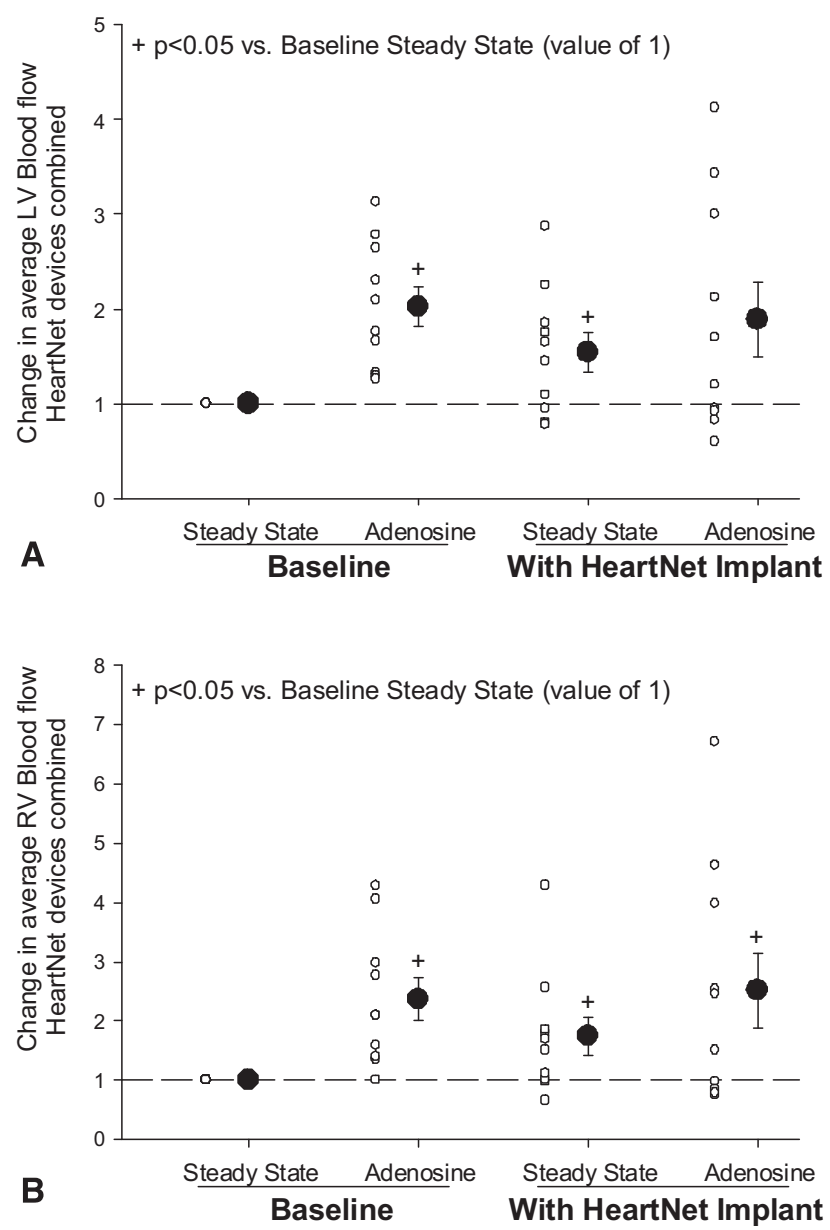

FIGURE 4. A, Change in average left ventricular $(L V)$ myocardial blood flow from normalized baseline steady state values. The LV myocardial blood flow values were increased under baseline adenosine and HeartNet Implant steady state conditions, yet failed to reach statistical significance under HeartNet Implant and adenosine condition. B, Change in average right ventricular (RV) myocardial blood flow from normalized baseline steady state values. RV myocardial blood flow values were elevated under baseline adenosine, HeartNet Implant steady state, and HeartNet Implant adenosine conditions. Sample size: $\mathrm{n}=10$ dilated cardiomyopathy (DCM) pigs. $+P<.05$ versus normalized baseline steady state value.

Through a mixed model analysis of variance, a significant interaction between the HeartNet Implant and adenosine infusion effects on LV myocardial blood flow was observed $(\mathrm{F}=6.21, P=.019)$. That is, the preparation with the HeartNet Implant placed showed a significant influence on adenosine-mediated myocardial blood flow, as compared with baseline. A regional response analysis was performed to determine whether the effect of acute HeartNet Implant placement on adenosine-mediated LV blood flow was global or regional (Figure 3). Before HeartNet Implant placement, all LV free wall regions demonstrated a consistent increase in blood flow with adenosine infusion. However, after acute HeartNet Implant placement, a selective negative myocardial blood flow response was observed in the LV free wall epicardial regions as compared with the respective endocardial regions. The LV free wall epicardial regions were more affected than the endocardial regions, with respect to HeartNet Implant placement and adenosine infusion $(P=.02)$.

The absolute change in LV and RV myocardial blood flow values as a function of steady state baseline values was computed, as shown in Figure 4. LV myocardial blood flow values after adenosine infusion and at steady state after HeartNet Implant placement were increased from steady state baseline. The LV myocardial blood flow after acute HeartNet Implant placement and adenosine infusion appeared elevated from baseline steady state but did not reach statistical significance. RV myocardial blood flow was elevated at all subsequent time points from normalized steady state baseline values.

\section{DISCUSSION}

The fundamental structural phenotype of DCM involves substantial LV chamber dilation and wall thinning, with an alteration in the volume/wall thickness ratio. Because the dilated ventricle is the cornerstone of DCM, attempts have been made to alter this inexorable disease course through physical support and restraint of the heart. 1-7,21-23 However, whether and to what degree myocardial blood flow patterns are acutely altered by HeartNet Implant placement in the context of DCM remains unknown. Accordingly, in the present study we evaluated 2 versions of the HeartNet Implant in a clinically relevant model of DCM to examine the effects of acute HeartNet Implant placement on hemodynamics and myocardial blood flow patterns. The pertinent findings of the study were 2-fold. First, placement of the HeartNet Implant did not acutely compromise hemodynamics in this well-established DCM model. Second, LV myocardial blood flow patterns were unchanged at steady state after HeartNet Implant placement. However, maximal coronary reserve was blunted, in absolute and relative terms, from steady state baseline after HeartNet Implant placement. Therefore, acute placement of the HeartNet Implant in the porcine DCM model had no negative steady state effects on hemodynamics or myocardial blood flow. However, it remains to be established whether and to what extent the acute effects on coronary reserve are operative after longer durations of deployment.

Several cardiac support devices have been developed with the goal of attenuating the progression of DCM. ${ }^{1-7,21-23}$ The CorCap Cardiac Support Device (Acorn Cardiovascular, Inc, St Paul, Minn) has been examined in several experimental models of heart failure and has advanced to clinical trials, demonstrating sustained reduction in LV end-diastolic and end-systolic volumes compared with controls. ${ }^{1}$ Whereas the CorCap device consists of a polyester-weave device that is placed around both ventricles, other devices have used a splint approach to cardiac support. 
Specifically, the Myosplint system (Myocor, Maple Grove, Minn) uses an implantable transventricular tension member fixed between epicardial pads to disperse epicardial tension. ${ }^{21}$ The Myosplint device has fallen out of clinical use owing to a lack of therapeutic effect and complications of mitral valve insufficiency. ${ }^{22}$ The Paracor HeartNet Implant has been examined in several animal models of LV failure and dysfunction. ${ }^{6,23}$ For example, Magovern and associates ${ }^{23}$ reported that long-term HeartNet implantation decreased postinfarction LV dilation in sheep. However, no preclinical studies examining myocardial blood flow in a DCM model have been undertaken, making the study described herein unique.

The present study used a well-characterized rapid pacing model that results in LV dilation and the hemodynamic phenotype of DCM. ${ }^{8-14}$ Acute HeartNet Implant placement in this DCM model did not create hemodynamic compromise. Taken together with the finding that the pulmonary capillary wedge pressure waveforms after placement of the HeartNet devices were similar to those recorded before device deployment, it is unlikely that acute placement of the device altered the degree of mitral regurgitation and/or had confounding influence on the LV myocardial blood flow measurements. Therefore, the myocardial blood flow measurements were performed under an equivalent hemodynamic state. After acute HeartNet Implant placement, LV myocardial blood flow was not compromised. In fact, there was a tendency for increased myocardial blood flow with acute HeartNet Implant placement. The reasons for this increase are likely multifactorial, including the surgical manipulation required to deploy the implant, disruptive electrical-mechanical events, neurohormonal stimulation, and localized sympathetic stimulation from the implant and the deployment procedure. Also, heart rate, a fundamental determinant of myocardial oxygen demand and myocardial blood flow, trended upward with HeartNet Implant placement. Whether these changes in myocardial blood flow and heart rate are maintained with long-term deployment of the HeartNet devices in the context of DCM remain to be established.

Maximal coronary flow reserve can be defined as the increase in coronary blood flow above basal flow values when the coronary vasculature is maximally dilated. ${ }^{24} \mathrm{Al}-$ though there are many ways to assess myocardial flow reserve, one common method is through the intravenous infusion of adenosine. ${ }^{24,25}$ In the present study, adenosine infusion robustly augmented myocardial blood flow from baseline steady state values, consistent with coronary vasodilatory effects. However, maximal LV coronary blood flow reserve, as defined by adenosine infusion compared with baseline conditions, was blunted by HeartNet Implant placement. Regional blood flow analysis revealed that the area most affected with respect to adenosine-mediated blood flow was the LV free wall epicardium. The reasons for the reduced adenosine-mediated coronary flow reserve in the LV free wall epicardial regions are likely multifactorial and could be due to mechanical or neurohormonal factors. One possible contributing factor could have been local sympathetic stimulation related to the placement of the HeartNet Implant, which may have subsequently altered G-protein coupled responses associated with the mechanism of action for adenosine. Additionally, an adenosine hysteresis effect may have occurred, decreasing the coronary response to the second adenosine infusion. Further studies using a cross-over design would be needed to investigate this possibility. The theoretical epicardial compressive force of the HeartNet Implant was approximately 0.5 to $2.4 \mathrm{~mm} \mathrm{Hg}$ as determined by echocardiographic data and intraoperative HeartNet Implant measurements. This level of epicardial pressure may have influenced coronary flow reserve given the elevated LV end-diastolic pressures and reduced LV wall thickness in the DCM model used. However, this potential mechanism remains speculative. Specifically, a recent report using finite element analysis modeling suggested that an epicardial compressive pressure of greater than $2.5 \mathrm{~mm} \mathrm{Hg}$ may negatively affect global LV systolic function (ie, cardiac output) ${ }^{26}$ However, in the present study, which used a physiologically and clinically relevant model and measurements, there was no compromise of LV systolic performance after acute HeartNet Implant placement. This underscores that theoretical effects must be carefully tested in relevant physiologic systems. Although multiple interventions, such as adenosine infusion ${ }^{9}$ or exercise, ${ }^{13,14}$ have been performed while measuring myocardial blood flow in this DCM model, the direct effects of ventricular elastic support have not previously been evaluated. Furthermore, an important caveat is that evaluation of maximal coronary flow reserve with adenosine infusion is more theoretical than practical ${ }^{24}$; therefore, whether and to what extent this blunting of coronary reserve holds clinical relevance is unclear.

Consistent with the clinical phenotype, the porcine DCM model causes biventricular failure. ${ }^{8,10-14}$ RV failure is of particular clinical relevance, and owing to the geometric and physiologic differences in this chamber, RV blood flow was measured. Steady state RV blood flow was unaffected by placement of the HeartNet Implant, and blood flow increased after adenosine infusion. Thus, RV blood flow patterns were unaffected by acute HeartNet Implant placement. It must be recognized that maximal coronary blood flow occurs at distinctly different points in the cardiac cycle for the $\mathrm{LV}$ and $\mathrm{RV}$, with the RV maximal coronary flow during systole and the LV maximal coronary flow during diastole. Therefore, these differential findings with respect to the coronary reserve in the LV and $\mathrm{RV}$ suggest that wall stress at end-diastole was maximized with the HeartNet devices at maximal stretch with minimal effects on wall stresses during systole. 
The present study was not without limitations. First, the HeartNet Implants were placed for the short-term, not the long-term, as in the clinical context. Second, although the rapid pacing-induced DCM model used in the present study recapitulates a severe clinical phenotype, rapid pacing is an unlikely cause for DCM in patients and therefore extrapolation to the clinical context must be done with caution. Third, the present study evaluated 2 different HeartNet Implants (models P140 and P150), by which the epicardial compressive forces were estimated but not directly computed using finite element analysis. Nevertheless, the unique findings from the present study demonstrated that in a porcine DCM model, the HeartNet Implant can be deployed without hemodynamic compromise. Although the HeartNet Implant did not affect myocardial blood flow under steady state conditions, a region-dependent decrease in LV free wall epicardial blood flow with respect to adenosine infusion was observed with acute HeartNet Implant placement. Future studies will need to address whether and to what degree this effect is present with long-term placement of the HeartNet Implant. Furthermore, the effects of HeartNet Implant placement on LV shape, size, and myocardial structure, as well as load-independent indices of LV function over a longer study period, remain to be established.

\section{References}

1. Starling RC, Jessup M, Oh JK, Sabbah HN, Acker MA, Mann DL, et al. Sustained benefits of the CorCap Cardiac Support Device on left ventricular remodeling: three year follow-up results from the Acorn clinical trial. Ann Thorac Surg. 2007;84:1236-42

2. Mann DL, Acker MA, Jessup M, Sabbah HN, Starling RC, Kubo SH. Clinical evaluation of the CorCap Cardiac Support Device in patients with dilated cardiomyopathy. Ann Thorac Surg. 2007;84:1226-35.

3. Acker MA, Bolling S, Shemin R, Kirklin J, Oh JK, Mann DL, et al. Mitral valve surgery in heart failure: insights from the Acorn Clinical Trial. J Thorac Cardiovasc Surg. 2006;132:568-77, 77.e1-4.

4. Blom AS, Mukherjee R, Pilla JJ, Lowry AS, Yarbrough WM, Mingoia JT, et al. Cardiac support device modifies left ventricular geometry and myocardial structure after myocardial infarction. Circulation. 2005;112: 1274-83.

5. Klodell CT Jr, Aranda JM Jr, McGiffin DC, Rayburn BK, Sun B, Abraham WT, et al. Worldwide surgical experience with the Paracor HeartNet cardiac restraint device. J Thorac Cardiovasc Surg. 2008;135:188-95.

6. Magovern JA. Experimental and clinical studies with the Paracor cardiac restraint device. Semin Thorac Cardiovasc Surg. 2005;17:364-8.

7. George I, Cheng Y, Yi GH, He KL, Li X, Oz MC, et al. Effect of passive cardiac containment on ventricular synchrony and cardiac function in awake dogs. Eur J Cardiothorac Surg. 2007;31:55-64.
8. Spinale FG, Hendrick DA, Crawford FA, Smith AC, Hamada Y, Carabello BA. Chronic supraventricular tachycardia causes ventricular dysfunction and subendocardial injury in swine. Am J Physiol. 1990;259(1 Pt 2):H218-29.

9. Spinale FG, Zellner JL, Tomita M, Tempel GE, Crawford FA, Zile MR. Tachycardia-induced cardiomyopathy: effects on blood flow and capillary structure. Am J Physiol. 1991;261(1 Pt 2):H140-8.

10. Spinale FG, Tanaka R, Crawford FA, Zile MR. Changes in myocardial blood flow during development of and recovery from tachycardia-induced cardiomyopathy. Circulation. 1992;85:717-29.

11. Kribbs SB, Merritt WM, Clair MJ, Krombach RS, Houck WV, Dodd MG, et al. Amlodipine monotherapy, angiotensin-converting enzyme inhibition, and combination therapy with pacing-induced heart failure. Hypertension. 1998;31:755-65.

12. Krombach RS, Clair MJ, Hendrick JW, Houck WV, Zellner JL, Kribbs SB, et al Angiotensin converting enzyme inhibition, AT1 receptor inhibition, and combination therapy with pacing induced heart failure: effects on left ventricular performance and regional blood flow patterns. Cardiovasc Res. 1998;38:631-45.

13. Clair MJ, Krombach RS, Hendrick JW, Houck WV, Hebbar L, Kribbs SB, et al AT1 angiotensin II receptor inhibition in pacing-induced heart failure: effects on left ventricular performance and regional blood flow patterns. J Card Fail. 1998; 4:311-23.

14. Caparas SN, Clair MJ, Krombach RS, Hendrick JW, Houck WV, Kribbs SB, et al Brain blood flow patterns after the development of congestive heart failure: ef fects of treadmill exercise. Crit Care Med. 2000;28:209-14.

15. Lucas DG Jr, Patterson T, Hendrick JW, Holder J, Sample JA, Joffs C, et al Effects of adenosine receptor subtype A1 on ventricular and renal function. J Cardiovasc Pharmacol. 2001;38:618-24.

16. Glenny RW, Bernard S, Brinkley M. Validation of fluorescent-labeled microspheres for measurement of regional organ perfusion. J Appl Physiol. 1993;74: 2585-97.

17. Prinzen FW, Glenny RW. Developments in non-radioactive microsphere techniques for blood flow measurement. Cardiovasc Res. 1994;28:1467-75.

18. Takagaki M, McCarthy PM, Goormastic M, Ochiai Y, Doi K, Kopcak MW Jr, et al. Determinants of the development of mitral regurgitation in pacinginduced heart failure. Circ J. 2003;67:78-82.

19. Akiyama M, Popovic ZB, Kamohara K, Cingoz F, Daimon M, Otaki C, et al. Acute reduction of functional mitral regurgitation in canine model using an epicardial device. Ann Thorac Surg. 2008;85:1771-5.

20. Maniu CV, Patel JB, Reuter DG, Meyer DM, Edwards WD, Rihal CS, et al. Acute and chronic reduction of functional mitral regurgitation in experimental heart failure by percutaneous mitral annuloplasty. J Am Coll Cardiol. 2004;44: 1652-61.

21. Schenk S, Reichenspurner H, Groezner JG, Boehm DH, Schirmer J, Scheidt WV, et al. Myosplint implantation and ventricular shape change in patients with dilative cardiomyopathy—first clinical experience. J Heart Lung Transplant. 2001; 20:217.

22. Christiansen S, Klocke A, Autschbach R. Past, present, and future of long-term mechanical cardiac support in adults. J Card Surg. 2008;23:664-76.

23. Magovern JA, Teekell-Taylor L, Mankad S, Dasika U, McGregor W, Biederman RW, et al. Effect of a flexible ventricular restraint device on cardiac remodeling after acute myocardial infarction. ASAIO J. 2006;52:196-200.

24. Marcus ML. The coronary circulation in health and disease. New York: McGraw Hill; 1983.

25. Berne RM. The role of adenosine in the regulation of coronary blood flow. Circ Res. 1980;47:807-13.

26. Jhun CS, Wenk JF, Zhang Z, Wall ST, Sun K, Sabbah HN, et al. Effect of adjustable passive constraint on the failing left ventricle: a finite-element model study. Ann Thorac Surg. 2010;89:132-7. 